\title{
Minimizing tissue damage due to filler injection with systemic hyperbaric oxygen therapy
}

\author{
Woo Taik Hong, \\ Jlye Kim, \\ Sug Won Kim \\ Department of Plastic and \\ Reconstructive Surgery, Wonju \\ Severance Christian Hospital, Yonsei \\ University Wonju College of Medicine, \\ Wonju, Korea
}

Recently, there is a growing interest of hyperbaric oxygen therapy in many fields of medicine. We had a 43-year-old female patient presented with severe necrosis of the nose, philtrum, and upper lip due to retrograde arterial occlusion after nasolabial fold hyaluronic acid filler injection. Our patient went through 43 sessions of systemic hyperbaric oxygen therapy from December 2, 2017 to January 18,2018 . We administered 2.8 atmosphere absolute (ATA) for 135 minutes in the first session and the remaining sessions consisted of 2.0 ATA for 110 minutes. In reporting this case, we wish to provide a warning regarding the latent risk of filler injections and share our experience about minimizing soft tissue damage in the early stages with systemic hyperbaric oxygen therapy.

Keywords: Complication / Hyperbaric oxygenation / Intra dermal injection

\section{INTRODUCTION}

The side effects of hyaluronic acid (HA) filler injections are a few of the most important issues in aesthetic medicine and wound management. Generally, HA filler injections are considered to be reliable and safe. Complications, such as erythema or ecchymosis, are usually mild and transient [1]. However, severe side effects such as skin necrosis, visual loss and stroke have been reported more than before as the number of the HA filler injection case grows [2]. Our institution's hyperbaric oxygen therapy (HBOT) center has more than 30 years of experience related to HBOT therapy and adopted a new 6-patient chamber (TPC-101; Ibex Medical Systems, Wonju, Korea) in 2016. Undersea and Hyperbaric Medical Society-certified medical personnel operate the chamber and our department often collaborates with them. Thus far, patients have little opportunity to obtain HBOT therapy after suffering from the complications of

\footnotetext{
Correspondence: Sug Won Kim

Department of Plastic and Reconstructive Surgery, Wonju Severance Christian

Hospital, Yonsei University Wonju College of Medicine, 20 Ilsan-ro, Wonju 26426,

Korea

E-mail: sugwonkim@yonsei.ac.kr

Received February 7, 2019 / Revised June 25, 2019 / Accepted July 10, 2019
}

HA filler injections in Korea. In this report, we wish to address this aforementioned limitation by presenting the case of a 43-year-old female patient who was treated by HBOT after suffering complications due to HA filler injections.

\section{CASE REPORT}

A 43-year-old female patient underwent an HA filler injection ( $2 \mathrm{~mL}$ in total) procedure for both nasolabial folds at a local aesthetic clinic (December 1, 2017). Immediately, the patient complained of severe pain and hypoesthesia and was treated with hyaluronidase injection 24 hours later. As there was no improvement of symptoms, she visited our emergency room and was admitted for the management of the side effects (December 2, 2017) (Fig. 1). Systemic HBOT was started immediately. We administered 2.8 ATA for 135 minutes for this first session. We continued HBOT until January 18, 2018 and the remaining 42 sessions consisted of 2.0 ATA for 110 minutes. In addition, oral aspirin and beraprost (Astellas Pharma Korea Inc., Seoul, Korea) and intravenous alprostadil alfadex (Dong-A ST, Seoul, Korea) was administered. Topical nitroglycerin and steroidal ointment was applied directly to the affected area daily. 
On external carotid angiography performed on December 6, 2017, right facial artery occlusion was observed and ipsilateral superior labial artery and angular artery could not be identified (Fig. 2). Experimental and clinical experiences suggest that HBOT reduces tissue loss by demarcating the border between necrotized and healthy tissues [3]. This helped us minimize the area of surgical removal in this case. Surgical debridement was done on December 20, 2017 (Fig. 3).

Retrospective studies have suggested that HBOT has synergy in combination with surgical debridement. There is no standard HBOT protocol for wound healing, and the guidelines for filler related vascular compromise are not concretely established. Initiating HBOT as soon as possible and continuing until the lesion improved is widely accepted principle [4]. However, since 2001, most of the clinicians who are interested in HBOT used 30 sessions of HBOT of 2-3 ATA through 5 or 6 weeks for humans [5]. We also targeted and utilized similar period and session number with those protocols. Our patient was hospitalized at our department for 30 days and underwent 27
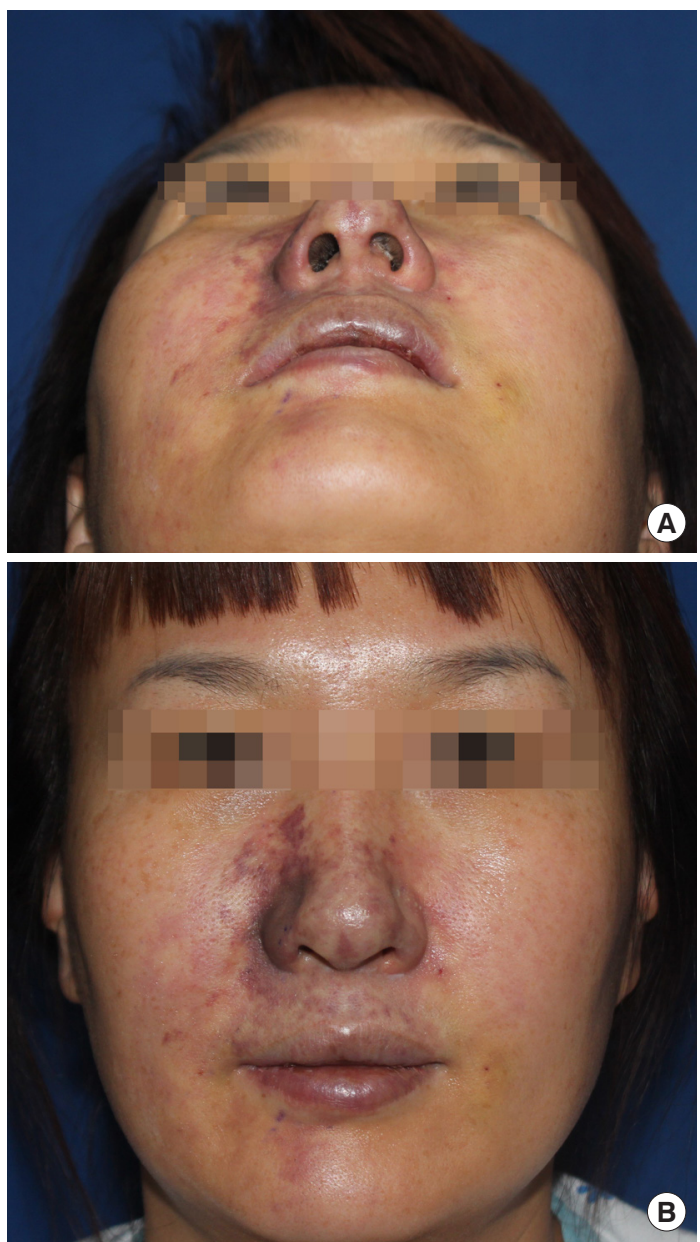

Fig. 1. Photographs of 24 hours after hyaluronic acid filler injection. (A) Submental view and (B) frontal view.

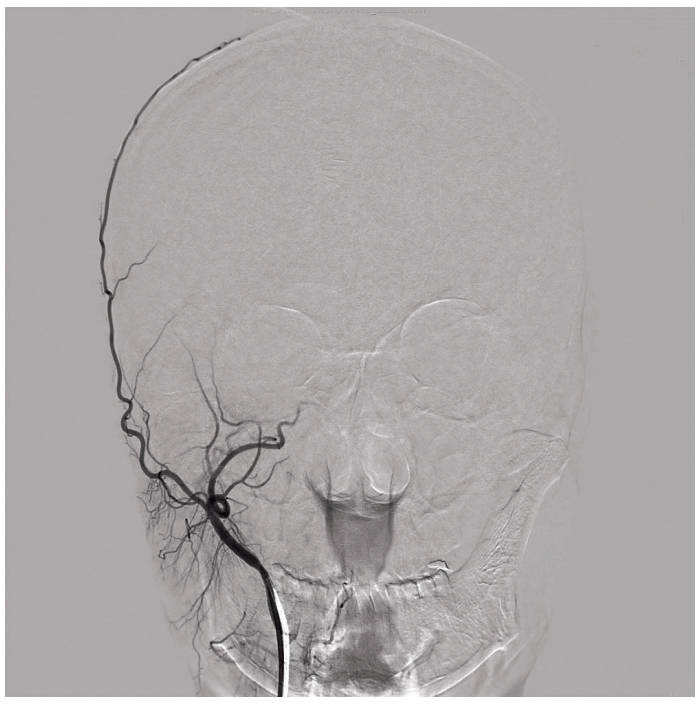

Fig. 2. External carotid angiography on 5 days after adverse event occurred.
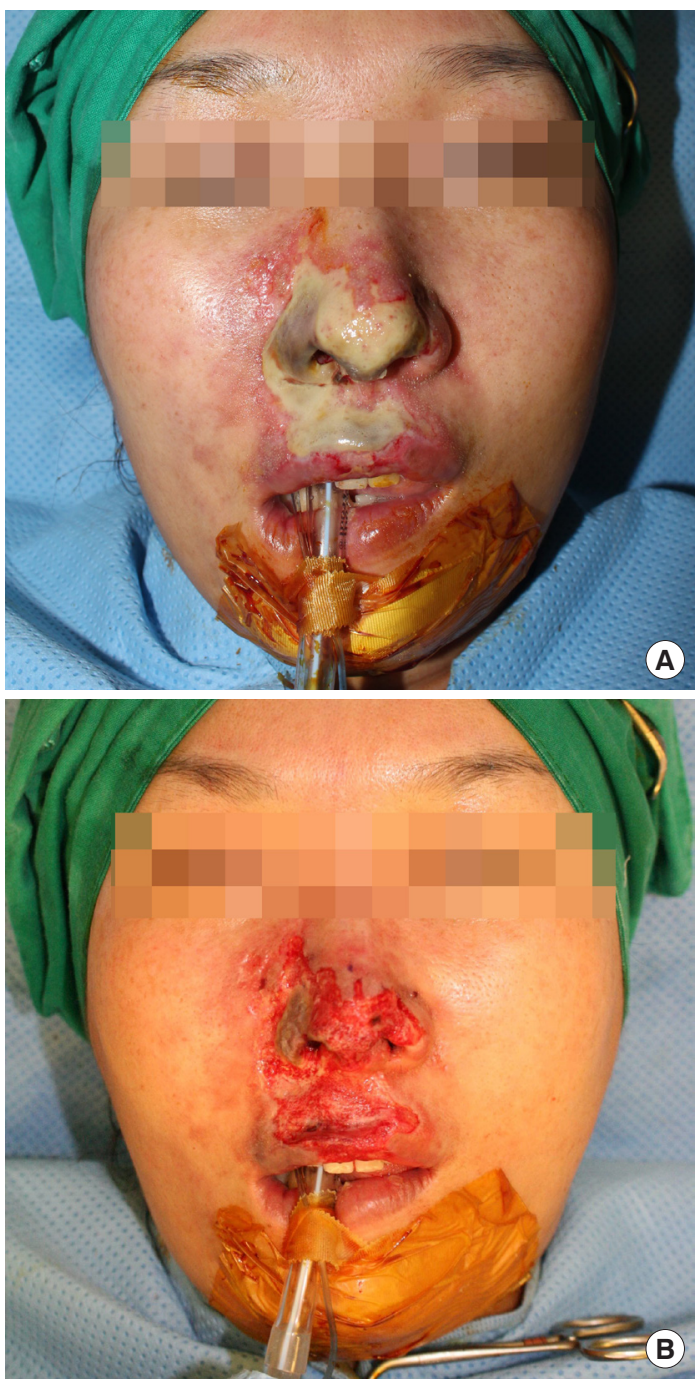

Fig. 3. (A, B) Debridement of necrotic region on 19 days after adverse event occurred. 

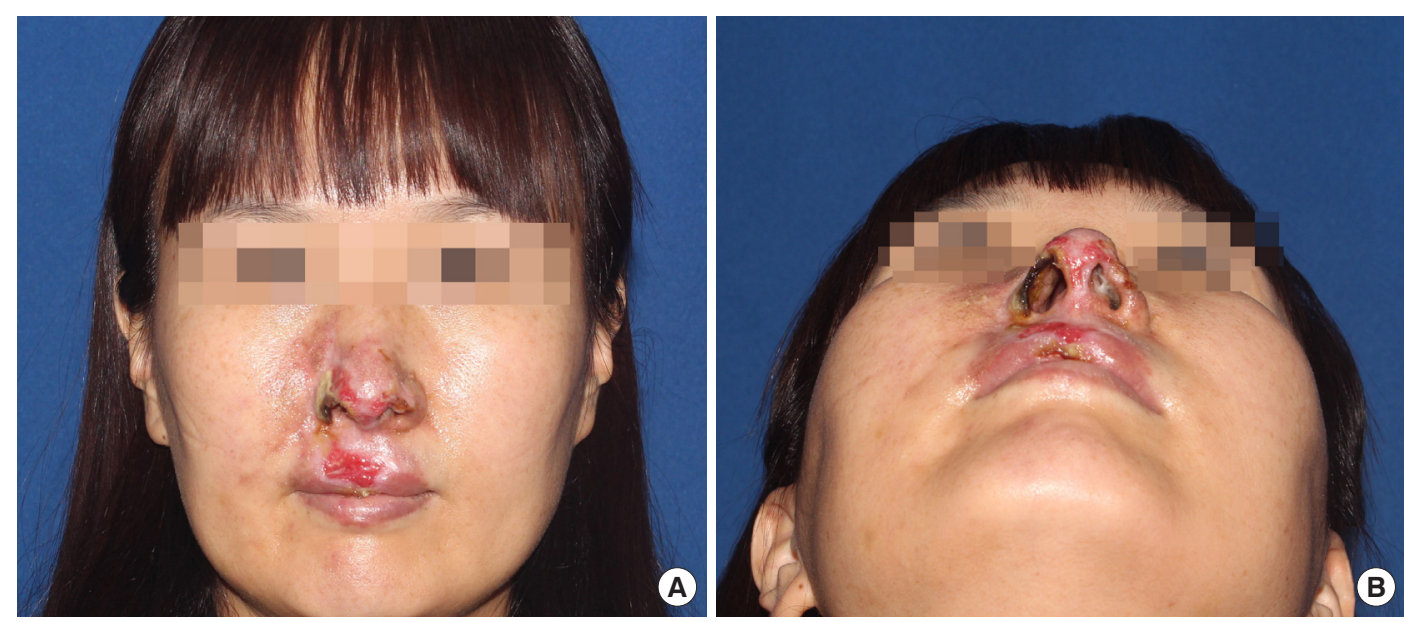

Fig. 4. Twenty-seven sessions of HBOT completed for 30 days from adverse event occurred. (A) Frontal view and (B) submental view.

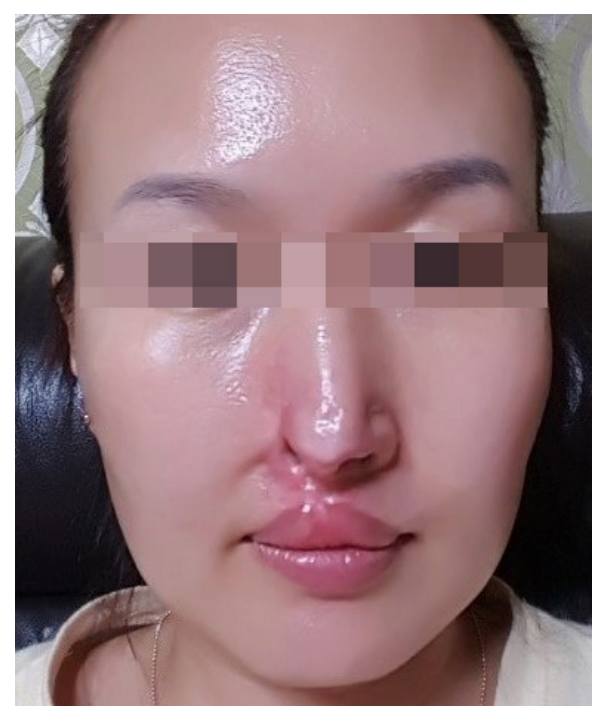

Fig. 5. Six months after the adverse event occurred.

sessions of HBOT during that period (Fig. 4). She was satisfied with the early treatment results and wanted to go through some more sessions after being discharged.

Considering her debridement date (December 20, 2017) and its maturation phase, we advised her to get another 15 sessions for 3 weeks at our emergency medicine outpatient clinic. In our institution, all patients who want to undergo HBOT as outpatients should be enrolled as emergency medicine patients for safety reasons. She underwent 16 more sessions (total 43 sessions, including hospitalization period sessions) for 16 days (total 46 days including hospitalization period). After that, she received sterilization treatment using anti-bacterial ointment and $2 \mathrm{~mm}$ foam for 2 weeks in the local clinic.

According to the patient, remnant wound fully healed in that period and there was no further change after that (Fig. 5). Although the treatment result was not perfect, patient was satis- fied with the fact that she was able to minimize the damage confined to right alar.

\section{DISCUSSION}

HA filler injection for augmentation is a popular procedure due to the simplicity of the technique and reasonable cost [6]. Not only the high compatibility with human connective tissue but also the possibility of reversing side effects with hyaluronidase made HA filler injection a favorite among many clinicians. However, as the number and extent of HA filler injections increase, the amount of complications also increases [7].

The most common side effects after HA filler injection are edema, pain, erythema, itching, and ecchymosis. Fortunately, most of these side effects are self-limited and last for no more than 2 weeks. Vascular occlusion is the most significant adverse event associated with HA filler injection. As in our case, this can be localized and result in skin necrosis, or distant, causing blindness or even cerebral ischemia.

The lower face, especially near the nose, is one of the areas most frequently injected with HA filler in the body. The descent of malar fat with age causes the nasolabial crease to deepen, and many patients ask for this to be corrected with HA filler. HA filler is also frequently used for smoothing an irregular nasal dorsum contour. These examples explain why so many of the complications occur around the nose recently.

However, the anatomical characteristics of the nose are also closely associated with these complications; therefore, a thorough understanding of the facial anatomy is very important. The nasal vessels run superficially, and clinicians should pay attention not only to a potential direct vessel injury but also to the filling effect of the HA filler itself. That is why aspiration before each injection and low injection pressure with minimal volume 
are recommended. Clinicians should give bolus injections only under the periosteal plane. An important landmark is located $2-3 \mathrm{~mm}$ superior of the alar groove because this is where the lateral nasal artery runs in the subdermal plexus [8]. Clinicians should stay above that plane when injecting HA filler into the lateral side of the nose.

It is important for physicians to prevent complications associated with HA filler injection with appropriate techniques [9]. Using a blunt cannula and mixing the HA with lidocaine or epinephrine may reduce the risk of complications. Avoiding previous scar regions and surgery sites is recommended [10].

However, eliminating all the complications of HA filler injection may be challenging. In such cases, minimizing the longterm sequelae is an important task for clinicians. Reducing excessive pressure in the affected compartment with anti-inflammatory effect of corticosteroids and increasing blood flow with nitroglycerin or aspirin can be helpful.

Nowadays, increasing the oxygen content with HBOT for wound management has been gaining interest worldwide and is reported as a useful tool for treating vascular compromise [4]. However, only a few cases with the potential to raise interest in HBOT have been reported so far [11]. The primary effect of HBOT is hyper-oxygenation increasing diffusion length and depth of oxygen through the tissues, thus resulting in anti-ischemic effect. Several studies have reported that HBOT not only helps mobilizing stem cells by enhancing their homing mechanism but also improves angiogenesis [12]. This can contribute to the increase of microcirculation in the damaged area and reduction of both edema and infection [13].

Previously hypoxic tissues can restore their bactericidal ability by restoring neutrophil-mediated phagocytosis. By preventing release of proteases and free radicals, which cause vasoconstriction, reperfusion injury could also be minimized [3].

Controversies about not only how long but also with what intensity HBOT should be maintained for wound healing, in general, are ongoing. Additionally, the guideline for filler vascular compromise has not established concretely. However, initiating it immediately after the adverse event and continuing until the lesion improved is mostly recommended. For necrotizing fasciitis, HBOT is usually maintained until spread of necrosis no more appears in previously debrided lesions. However, since 2001, many researches follow the protocol of 30 sessions of 2-3 ATA through 5 or 6 weeks [5].

In the present case, we treated this patient with 43 sessions of HBOT to minimize soft tissue damage at an early stage (until 46 days from the first adverse event). In view of our experience with HBOT, it is difficult to expect complete functional or cosmetic recovery by HBOT alone. However, it seems to have a good effect in reducing the necrosis range. In reporting this case, we wish to provide a warning regarding the latent risk of filler injections, and report that soft tissue damage in the early stages can be minimized with systemic HBOT.

\section{NOTES}

\section{Conflict of interest}

No potential conflict of interest relevant to this article was reported.

\section{Ethical approval}

The study was approved by the Institutional Review Board of Wonju Severance Christian Hospital (IRB No. 2019-01-0049) and performed in accordance with the principles of the Declaration of Helsinki. Written informed consent was obtained.

\section{Patient consent}

The patient provided written informed consent for the publication and the use of her images.

\section{ORCID}

Woo Taik Hong https://orcid.org/0000-0002-6764-8755

Jlye Kim https://orcid.org/0000-0002-1724-4250

Sug Won Kim https://orcid.org/0000-0002-2354-7100

\section{REFERENCES}

1. Loh KT, Chua JJ, Lee HM, Lim JT, Chuah G, Yim B, et al. Prevention and management of vision loss relating to facial filler injections. Singapore Med J 2016;57:438-43.

2. Bertl K, Gotfredsen K, Jensen SS, Bruckmann C, Stavropoulos A. Adverse reaction after hyaluronan injection for minimally invasive papilla volume augmentation: a report on two cases. Clin Oral Implants Res 2017;28:871-6.

3. Leach RM, Rees PJ, Wilmshurst P. Hyperbaric oxygen therapy. BMJ 1998;317:1140-3.

4. Beer K, Downie J, Beer J. A treatment protocol for vascular occlusion from particulate soft tissue augmentation. J Clin Aesthet Dermatol 2012;5:44-7.

5. Medical Advisory Secretariat. Hyperbaric oxygen therapy for non-healing ulcers in diabetes mellitus: an evidence-based analysis. Ont Health Technol Assess Ser 2005;5:1-28.

6. Kim JE, Sykes JM. Hyaluronic acid fillers: history and overview. Facial Plast Surg 2011;27:523-8.

7. Kim JH, Ahn DK, Jeong HS, Suh IS. Treatment algorithm of complications after filler injection: based on wound healing process. J Korean Med Sci 2014;29(Suppl 3):S176-82. 
8. Rohrich RJ, Gunter JP, Friedman RM. Nasal tip blood supply: an anatomic study validating the safety of the transcolumellar incision in rhinoplasty. Plast Reconstr Surg 1995;95:795-9.

9. Bailey SH, Cohen JL, Kenkel JM. Etiology, prevention, and treatment of dermal filler complications. Aesthet Surg J 2011; 31:110-21.

10. Humphrey CD, Arkins JP, Dayan SH. Soft tissue fillers in the nose. Aesthet Surg J 2009;29:477-84.

11. Henderson R, Reilly DA, Cooper JS. Hyperbaric oxygen for ischemia due to injection of cosmetic fillers: case report and issues. Plast Reconstr Surg Glob Open 2018;6:e1618.

12. Pena-Villalobos I, Casanova-Maldonado I, Lois P, Prieto C, Pizarro C, Lattus J, et al. Hyperbaric oxygen increases stem cell proliferation, angiogenesis and wound-healing ability of WJMSCs in diabetic mice. Front Physiol 2018;9:995.

13. Chander Y, Misra RN, Rai R. Hyperbaric oxygen therapy [HBOT]. Med J Armed Forces India 1999;55:89-90. 